\title{
O IMPACTO DOS INDICADORES PIB PER CAPTA E ÍNDICE DE GINI NA ESPERANÇA DE VIDA DO BRASILEIRO: UMA PERSPECTIVA DO BRASIL E REGIÕES.
}

\author{
Cleydner Marques de Magalhães Maurício - Mestre em Administração pela UFAL \\ Professor de Economia da FEAC/UFAL \\ Fernando Sarmento Plech Filho - Graduado em Economia pela UFAL
}

\begin{abstract}
RESUMO - O trabalho tem como objetivo verificar a existência de correlação entre as variáveis como PIB per capita e coeficiente de Gini, com a expectativa de vida, tanto no Brasil como nas regiões brasileiras. Para que esse objetivo fosse alcançado, iniciamos com uma pesquisa bibliográfica, em seguida, por meio do software Excel, efetuamos a correlação das variáveis e estabelecemos um modelo de regressão. Os resultados obtidos comprovam que as variáveis independentes: Índice de Gini e PIB per capita, explicam o comportamento da Expectativa de vida. Isso comprova, que tanto no Brasil como em suas regiões, o PIB per capita e Índice de Gini aumentam a esperança de vida da população brasileira.
\end{abstract}

Palavras-chave: Esperança de Vida; Desigualdade; Indicadores; PIB.

\section{THE IMPACT OF GDP PER CAPITA INDICATORS AND GINI INDEX ON BRAZILIAN LIFE EXPECTANCY}

\begin{abstract}
The objective of this study was to verify the existence of a correlation between variables such GDP per capita and Gini Coeficient, with life expectation in Brazil and its regions. In order to achieve this goal, we started with a bibliographical research, then through Excel, we calculated the correlation index and established a regression model. The results afirms that the independent variables: Gini index and GDP per capita, explain the behaviour of Life Expectancy, which proves that in Brazil and in its regions GDP per capita and Gini Index increase the life expectancy of the Brazilian population.
\end{abstract}

Key-words: Expectancy of life; Social Inequality; Indicators; GDP.

\section{INTRODUÇÃO}

A expectativa de vida tem se revelado um bom parâmetro para avaliar em que nível de desenvolvimento se encontra um país. A melhora da expectativa de vida das pessoas possivelmente está ligada ao crescimento econômico, ao desenvolvimento social e a uma melhora na distribuição da renda. Os dados obtidos nessa pesquisa revelam que as regiões com renda maior tendem a uma expectativa de vida maior, enquanto que as regiões com renda menor levam a índices mais baixos na expectativa de vida.

Fatores históricos de ocupação e desenvolvimento provavelmente contribuíram para uma distribuição desigual de renda entre as regiões brasileiras e isso se refletiu nas condições de saúde e bem-estar das pessoas e consequentemente na esperança de vida de suas populações. No Brasil as disparidades regionais em parâmetros como PIB per capita e Índice de Gini e em variáveis 
correlacionadas tais como: gasto público em saúde, educação entre outras, evidencia a necessidade de políticas de desenvolvimento voltadas para a correção desses distúrbios históricos.

O artigo é de fundamental importância, uma vez que estabelece a correlação entre a variável dependente - Expectativa de Vida e as variáveis independentes: PIB per capita e Índice de Gini, no Brasil e nas cinco regiões brasileiras. Tem como objetivo geral verificar o comportamento da Expectativa de Vida nas regiões brasileiras e no Brasil, no período de 2000 a 2011, e a existência de correlação entre as variáveis acima citadas.

A hipótese construída é que existe uma correlação positiva quando associamos Expectativa de Vida ao PIB per capita, e negativa para: Expectativa de Vida e Índice de Gini. Essa correlação deverá ocorrer tanto no Brasil como nas regiões brasileiras, explicada por vários motivos, dentre eles que ao aumentar a renda per capita, haverá melhores condições de vida, possibilitando o acesso a uma boa alimentação e aos serviços de qualidade. E a redução das desigualdades possibilita uma maior expectativa de vida na população, justificando que quanto mais próximo a 0 (zero) melhor será a esperança de vida da população.

Do ponto de vista metodológico este estudo baseia-se em pesquisas bibliográficas e documentais onde é feita uma análise de dados de indicadores de desenvolvimento econômico e sociais correlacionados a expectativas de vida dos brasileiros no período de 2000 a 2011 . As análises e reflexões foram obtidas em função dos arquivos no banco de dados do IBGE e IPEADATA.

\section{EXPECTATIVA DE VIDA: BREVES CONSIDERAÇÕES.}

A expectativa de vida, indicador retirado das tábuas de mortalidade para saber qual a chance de vida que uma pessoa tem, ao nascer, por faixa etária até os 80 ou mais, está diretamente ligada às condições de saúde e vida das pessoas.

O cientista que tentou pela primeira vez construir uma tábua de mortalidade foi o inglês John Graunt que durante sua vida dedicou-se a coletar dados referentes à mortalidade das pessoas.

De acordo com Ferreira, (1985) apud Silva, L., (2008) "as observações de John Graunt publicadas em 1662 constituíram o primeiro exemplo do "método estatístico" e fizeram com que lhe desse o título de "Inventor da Ciência Atuarial".

A literatura afirma que houve, nesse período, a necessidade de controle populacional e de se obter dados estatísticos para os grandes centros urbanos, surgiu então à necessidade de se avaliar o tempo médio de vida das pessoas.

Para Silva, L., (2008) essa primeira pesquisa trazia resultados estatísticos para uma pequena população 
"Considerando que para 100 indivíduos nascidos vivos, cerca de 3 deles". morreram antes mesmo de completar de 6 (seis) anos de idade, e que apenas um sobreviveu até os 76 (setenta e seis) anos. Temos sete décadas entre 6 (seis) anos e 76 (setenta e seis) anos, e procuramos seis números médios proporcionais de óbitos entre os 64 indivíduos sobreviventes na idade de 6 (seis) anos e o único indivíduo que sobreviveu até os 76 (setenta e seis). Descobrimos que esses números médios proporcionais de óbitos são muito próximos da realidade, apesar dos seres humanos não morrerem em percentagem exata, nem em frações.” (GRAUNT, 1662, p.69 apud SILVA, L., 2008, tradução do autor)

Vários outros pesquisadores surgiram depois dos trabalhos de Graunt, a preocupação com o controle das mortes entre as populações se espalhou com o passar dos anos e com o desenvolvimento das cidades. Silva, L., (2008), destaca ainda a importância que teve:

O trabalho de Graunt, considerado por muitos pesquisadores como o pai da Ciência Atuarial, serviu como estímulo para uma série de outros trabalhos na pesquisa das tábuas de sobrevivência, com destaque para as tábuas de vida de Halley, publicadas pelo astrônomo inglês Edmond Halley (o pai do famoso cometa) em 1693,para o trabalho do francês Abraham de Moivre (1725), dos ingleses Joshua Milani (1815) e Benjamim Gompertz (1825) e também o estudo de William Makeham (1860), dentre outros.(SILVA, L., 2008, tradução do autor)

As tábuas de mortalidade no Brasil foram construídas com muito atraso em relação aos outros países. Segundo Giovanella et al (2012) "a esperança de vida em 1910 era de apenas 34,6 anos para a população feminina e 33,4 para a masculina."

As primeiras tábuas de mortalidade do Brasil foram construídas a partir de 1980 quando a primeira delas foi calculada com base nos resultados do Censo Demográfico daquele ano. Outras duas tábuas de mortalidade surgiram depois no censo 1991 e 2000 (IBGE, 2000)

De acordo com informações de publicações do IBGE (2010), com passar dos anos houve uma mudança, hoje:

A tábua de mortalidade divulgada anualmente, apresenta a expectativa de vida às idades exatas até os 80 anos. O modelo demográfico descreve o crescimento do volume populacional na composição etária da população entre a mortalidade e a fecundidade, bem como também é utilizada pelo Ministério da Previdência Social (MPS) como um dos parâmetros necessários para o cálculo das aposentadorias dos trabalhadores que estão sob o Regime Geral de Previdência. (IBGE, p.05, 2010)

Estudos revelam que houve uma mudança significativa nas condições de saúde da maior parcela da população brasileira que antes era rural, mas com o desenvolvimento e crescimento econômico, resultante de uma urbanização e industrialização das regiões como um todo, acelerou o processo de migração do campo para cidade.

Pesquisas vêm associando a relação entre crescimento econômico, desenvolvimento social e expansão das melhorias na saúde, ao aumento da expectativa de vida, principalmente quando se observa as variáveis que são importantes como: a educação (combate ao analfabetismo), o gasto público em saúde (melhoria da assistência médica).

Conforme Santos; Pales; Rodrigues, (2013, p.16), vários fatores contribuíram com o 
aumento da esperança de vida ao nascer o cenário brasileiro, dentre eles, o aumento da renda, da escolaridade, da assistência à saúde, saneamento básico e outros. Contudo, a melhora desses indicadores vem transformando o perfil etário da população que está envelhecendo.

Os dados sobre o estado e as condições de saúde da população brasileira, são determinados pela esperança de vida, uma estimativa proveniente da Pesquisa Nacional por Amostra de Domicílios (Pnad) do Instituto Brasileiro de Geografia e Estatística (IBGE), um indicador tradicional que pode ser tomado como indicador global de saúde.

A literatura demonstra que a esperança de vida ao nascer, de maneira geral, é mais baixa nos países africanos e asiáticos, apresenta valores intermediários nos países americanos e valores mais altos nos países europeus e na Oceania.

O Japão, com o maior valor mundial para a esperança de vida semelhante à dos países europeus, existem alguns casos que a esperança de vida ao nascer não é diretamente proporcional à riqueza do país, observa-se padrões diferenciados entre os países. Os Estados Unidos, país mais rico do mundo, têm esperança de vida próxima à observada em países relativamente pobres, como a Grécia. Giovanella, et al (2012).Os distintos graus de desenvolvimento econômico e social, que compõem a faixa etária da população e a distribuição de renda existente em cada região, podem ser observados por este indicador. Em Conformidade com as pesquisas de Thomas e Frankenberg, (2002) apud Pelegrini; Castro, (2012)

[...] discutem o papel da nutrição e da saúde para a produtividade, reforçando as evidências de que há uma forte associação entre melhores condições de saúde e crescimento econômico. No entanto, consideram que o nexo causal possa ser em ambas as direções, ou seja, com maior rendimento os indivíduos investem mais em capital humano, ou seja, em melhores dietas, saneamento e cuidados de saúde. E, por outro lado, sendo o trabalhador mais saudável e menos suscetível às doenças, é mais produtivo e obtém rendimento maior. (Thomas e Frankenberg, 2002 apud PELEGRINI; CASTRO, 2012, p.98)

Ao se avaliar as condições de saúde da população brasileira usando apenas este indicador, poder-se-ia dizer que as condições são regulares ou ruins, pois $18,92 \%$ a 3,8\% das pessoas declaram ter saúde regular, ruim ou muito ruim, o que é um percentual alto. Existem também diferenças na avaliação do estado de saúde segundo as regiões brasileiras, que, por sua vez, refletem os distintos graus de desenvolvimento econômico e social, a composição etária da população e a distribuição de renda existente em cada região.

No Brasil, a melhor situação é observada na região Sudeste, onde $80 \%$ das pessoas classificam sua saúde como boa ou muito boa. As regiões Sul e Centro-Oeste apresentam proporção um pouco menor de indivíduos com saúde boa ou muito boa (78\%). As piores condições são observadas nas regiões Norte e Nordeste, onde 76\% e 73\% dos indivíduos, respectivamente, consideram sua saúde boa ou muito boa conforme dados do (IBGE, 2008). As 
diferenças regionais em relação à esperança de vida podem ser constatadas desde as primeiras décadas do século passado, e refletem em níveis de sobrevivência discrepantes das populações, de cada uma das macrorregiões brasileiras nesse período.

Durante o período de 1960 a 2011 houve uma redução significativa nos padrões históricos das desigualdades regionais em relação à expectativa de vida. Segundo dados do IBGE a uma diferença bastante significativa na região Nordeste, entre os anos de 1960 e 2011, a diferença na expectativa de vida entre o Nordeste e Sul no período era de 13 anos, em 2011, caí para menos de 5 anos de idade. As diferenças entre as regiões no período de 2000 a 2011 , estão em torno de 4 e 5 anos aproximadamente.

Tabela 1: Esperança de Vida ao Nascer, 2000 - 2011.

\begin{tabular}{|c|c|c|c|c|c|c|c|c|c|c|c|c|}
\hline \multicolumn{10}{c|}{ Esperança de Vida ao Nascer do Brasil e Regiões } \\
\hline & 2000 & 2001 & 2002 & 2003 & 2004 & 2005 & 2006 & 2007 & 2008 & 2009 & 2010 & 2011 \\
\hline Brasil & 69,8 & 70,3 & 70,7 & 71,2 & 71,6 & 72 & 72,4 & 72,8 & 73,1 & 73,5 & 73,9 & 74,2 \\
\hline Norte & 67,9 & 68,2 & 68,5 & 68,8 & 69,1 & 69,4 & 69,7 & 70 & 70,3 & 70,5 & 70,8 & 71 \\
\hline Nordeste & 67,3 & 67,8 & 68,2 & 68,6 & 69 & 69,4 & 69,8 & 70,2 & 70,5 & 70,9 & 71,2 & 71,6 \\
\hline Sudeste & 71,1 & 71,7 & 72,1 & 72,6 & 73,1 & 73,5 & 74 & 74,4 & 74,8 & 75,2 & 75,5 & 75,9 \\
\hline Sul & 71,9 & 72,3 & 72,3 & 73,2 & 73,6 & 74 & 74,4 & 74,8 & 75,2 & 75,5 & 75,9 & 76,2 \\
\hline C. Oeste & 70,8 & 71,1 & 71,5 & 71,8 & 72,1 & 72,3 & 72,6 & 72,9 & 73,2 & 73,4 & 73,7 & 73 \\
\hline
\end{tabular}

Fonte: IBGE, Projeção da População do Brasil, Idade, para o Período 2000/2060.

Em geral, a esperança de vida ao nascer no país aumentou em todas as regiões, o ganho em anos no país no período 2000/20111, foi de 4,5 anos de idade o que dá em média 3 meses e 22 dias por ano. A região Sul é a que tem a melhor esperança de vida com 76,2 anos de idade, o Sudeste como região mais rica do país obteve o maior aumento entre todas as regiões para o período 2000-2011 com um ganho de 4,66 anos de idade, apesar do aumento no período o Sudeste fica em segundo com 75,9 anos de idade.

Tabela 2: Aumento da Esperança de Vida ao Nascer.

\begin{tabular}{l|ll|l}
\hline \multicolumn{3}{l}{ Aumento em Anos } \\
\hline & 2000 & 2006 & 2000 \\
Brasil & 2005 & 2011 & 2011 \\
Norte & 1,33 & 1,66 & 4,5 \\
Nordeste & 2,08 & 1,41 & 3,25 \\
Sudeste & 2,41 & 1,66 & 4,25 \\
Sul & 2,08 & 1,66 & 4,66 \\
C. Oeste & 1,41 & 1,16 & 3,08 \\
\hline \multicolumn{4}{r}{ Fonte: } \\
\hline
\end{tabular}


O Nordeste sempre apresentou os piores indicadores, e o da expectativa de vida não é diferente, mas nas últimas décadas vêm melhorando sua posição na esperança de vida ao nascer.

A região apresentou no ano 2000 o pior resultado entre as regiões 67,3 anos de idade, já no ano de 2011, o Nordeste tem uma melhora, ficando à frente da região Norte, com 71,6 anos de idade, isso demonstra o grau de evolução econômica e social da região.

\section{METODOLOGIA}

A pesquisa quantitativa analisou indicadores econômicos relativos ao período proposto e foram obtidas informações dos arquivos do banco de dados encontrados no Sidra, site do Instituto Brasileiro de Geografia e Estatística (IBGE) e no site do Instituto de Pesquisa Econômico Aplicada - IPEADATA.

A análise qualitativa contou com uma pesquisa bibliográfica onde foi possível observar as diferenças regionais, os aspectos de desenvolvimento econômico e social e seu reflexo na expectativa de vida do brasileiro. Para a análise dos dados será utilizado o modelo econométrico simples, de regressão linear. Este modelo, consistirá na obtenção de uma equação explicativa entre as variáveis, pelo método ANOVA (análise de variância). Os resultados para os testes de analise representarão se os fatores são significativos para um bom modelo de regressão linear.

Em economia é comum efetuar a correlação entre as variáveis. De posse dos dados brutos, é possível organizar e transformar informações relevantes para que possam subsidiar tomadas de decisões. Para verificar se há alguma correlação entre a Expectativa de Vida (variável dependente) e as variáveis: PIB per capita e o Coeficiente de Gini (variáveis independentes), faremos uso do coeficiente de correlação de Pearson (r).

De acordo com Pocinho, (2009) O coeficiente de correlação pode ser interpretado com base em:

$\mathrm{r} \leq|0,2| \quad$ Correlação muito baixa (valores desprezíveis)

$|0,2|<\mathrm{r} \leq|0,5| \quad$ Correlação baixa

$|0,5|<\mathrm{r} \leq|0,7| \quad$ Valores significativos

$|0,7|<\mathrm{r} \leq|0,9| \quad$ Correlação Alta

$|0,9|<\mathrm{r} \leq|1| \quad$ Correlação muito alta

Já o coeficiente de determinação $\mathrm{R}^{2}$ varia de 0 a 1 estabelecendo quanto da variação da variável Y é explicada pela variável independente.

Dessa forma o artigo apresenta-se dirigido pelas hipóteses

\section{- Hipótese Nula (ho)}

$h_{0}$ : A Expectativa de Vida tem correlação com as variáveis: PIB per capita e Índice de Gini. 
- Hipótese Alternativa $\left(h_{1}\right)$

$\mathrm{h}_{1}$ : Pode se construir um modelo de regressão, capaz de estimar a Expectativa de Vida.

\section{Análise de Regressão}

Para construção do modelo foram utilizadas as variáveis:

- Índice de Gini

- $\quad$ PIB per capita

Em seguida, o modelo será testado, averiguando-se: Coeficiente de determinação ou de explicação $-\boldsymbol{R}^{2}$; estatística $\boldsymbol{F}$, estatística $\boldsymbol{t}$ para os coeficientes das variáveis explicativas e os sinais dos coeficientes das variáveis independentes.

\section{RESULTADOS E DISCUSSÕES.}

\section{Expectativas de Vida $x$ PIB per capita e Índice de Gini}

Os resultados apresentados nos quadros que seguem, mostram que tanto na analise para o Brasil, como para as regiões (Norte, Nordeste, Centro-Oeste, Sul e Sudeste) verifica-se a existência uma forte correlação positiva entre Expectativa de Vida e PIB per capita, cujos valores variaram entre 0,926 e 0,988 . Esses valores encontram-se dentro do esperado, uma vez que quanto maior for o PIB per capita, maior o poder de compra, ceteres paribus. O PIB per capita não é um dado ideal, mas países com maior PIB per capita tendem a ter maior Índice de Desenvolvimento Humano (ID).

Quadro 1 Correlação de Person -BRASIL

\begin{tabular}{|c|c|}
\hline \hline Matriz de & Correlação \\
\cline { 2 - 2 } Correlação & Expectativa de Vida \\
Expectativa de Vida & 1 \\
\hline PIB per capita & 0,952024281 \\
Coeficiente de Gini & $-0,994131497$ \\
\hline \hline
\end{tabular}

Quadro 2 Correlação de Person: Regiões (Norte, Nordeste, Sudeste, Sul, centro Oeste).

\begin{tabular}{|l|l|l|}
\hline REGIÕES & Expectativa de Vida & 1 \\
\hline \multirow{4}{*}{ Norte } & PIB per capita & 0,9888868 \\
\cline { 2 - 3 } & Coeficiente de Gini & $-0,7479174$ \\
\hline \multirow{3}{*}{ Nordeste } & Expectativa de Vida & 1 \\
\cline { 2 - 3 } & PIB per capita & 0,972000 \\
\cline { 2 - 3 } & Coeficiente de Gini & $-0,987698$ \\
\hline \multirow{3}{*}{ Sudeste } & Expectativa de Vida & 1 \\
\cline { 2 - 3 } & PIB per capita & 0,926453 \\
\cline { 2 - 3 } & Coeficiente de Gini & $-0,917059$ \\
\hline \multirow{3}{*}{ sul } & Expectativa de Vida & 1 \\
\cline { 2 - 3 } & PIB per capita & 0,938257 \\
\hline
\end{tabular}




\begin{tabular}{|l|l|l|}
\hline & Coeficiente de Gini & $-0,909864$ \\
\hline \multirow{3}{*}{ C. Oeste } & Expectativa de Vida & 1 \\
\cline { 2 - 3 } & PIB per capita & 0,981921 \\
\cline { 2 - 3 } & Coeficiente de Gini & $-0,94442$ \\
\hline
\end{tabular}

Quanto à correlação entre a Expectativa de Vida e o Coeficiente de Gini, que varia entre 0 e 1 (quanto mais próximo a 0 haverá uma completa igualdade na renda), todos apresentaram uma boa correlação negativa, pois mais próximo a 0, implicaria em uma maior Expectativa de Vida. A região Norte foi a que apresentou um Coeficiente de Gini mais distorcido, - 0,747 se comparado com os das outras regiões e do Brasil, que variaram entre $(-0,909$ a -0,994), conforme quadro acima.

Após o estudo da correlação, em que foi possível estabelecer uma excelente correlação positiva entre a Expectativa de Vida e o PIB per capita, bem como uma ótima correlação negativa entre a Expectativa de Vida e o Coeficiente de Gini, procurou-se analisar o comportamento da variável dependente - Expectativa de Vida (EV) em relação ao PIB per capita (PIBpc) e ao coeficiente de Gini (CGini) variável independente.

Num primeiro momento foram analisados os dados do Brasil, relacionando a EV com o PIBpc. Em seguida, foi feita a corelação da EV com o C.Gini.

Da mesma forma que a análise foi realizada para o Brasil, conforme detalhamento no parágrafo anterior procedeu-se o estudo para as 5 (cinco) regiões brasileiras: Norte $(\mathrm{N})$, Nordeste (NE), Centro-Oeste (CO) Sul (S) e Sudeste (SE).

O intuito da análise é conhecer o comportamento da EV no Brasil e em suas regiões, bem como estabelecer as correlações e suas intensidades com as variáveis PIBpc e CGini.

A escolha da variável PIBpc refere-se à necessidade de conhecer como as regiões brasileiras se comportaram no período em análise, no tocante a como indivíduos se beneficiariam de um incremento na produção.

Entretanto, vale ressaltar que o PIB per capita não é uma medida de renda pessoal. Ele pode aumentar enquanto a maioria das pessoas fica mais pobres, ou proporcionalmente não tão ricas, uma vez que o PIB não considera o nível de desigualdade de renda de uma sociedade.

Para complementar a análise, o CGini, foi escolhido, objetivando verificar se ocorreu uma maior ou menor concentração de renda nas regiões analisadas. Justifica-se a escolha por ser o Coeficiente de Gini (CGini) uma medida estatística de desigualdade, usada para indicar o grau de concentração de renda de uma região. 


\section{BRASIL EXPECTATIVADE VIDA (E.V.) X PIB PER CAPITA (PIBPC)}

Em uma etapa inicial foram dispostos os dados da tabela (1) Expectativa de vida e PIB per capita tabela (11) abaixo, que resultou em um gráfico utilizando a planilha do software Excel, pode-se observar a função gerada, conforme gráfico abaixo.

Tabela 3 PIB per capita: 2000 - 2011

\begin{tabular}{l|l|ll|ll|l}
\hline & Brasil & Norte & Nordeste & Sudeste & Sul & C. Oeste \\
\hline $\mathbf{2 0 0 0}$ & 15,44 & 9,31 & 7,16 & 20,53 & 16,14 & 18,66 \\
$\mathbf{2 0 0 1}$ & 15,41 & 9,39 & 7,13 & 20,31 & 18,42 & 19,04 \\
$\mathbf{2 0 0 2}$ & 15,60 & 9,63 & 7,25 & 20,49 & 18,53 & 19,51 \\
$\mathbf{2 0 0 3}$ & 15,55 & 9,97 & 7,29 & 20,17 & 18,74 & 19,89 \\
$\mathbf{2 0 0 4}$ & 16,20 & 10,57 & 7,67 & 20,96 & 19,41 & 20,67 \\
$\mathbf{2 0 0 5}$ & 16,47 & 11,01 & 7,93 & 21,39 & 19,02 & 21,22 \\
$\mathbf{2 0 0 6}$ & 16,89 & 11,30 & 8,21 & 21,96 & 19,39 & 21,45 \\
$\mathbf{2 0 0 7}$ & 18,19 & 12,04 & 8,62 & 23,86 & 21,09 & 22,98 \\
$\mathbf{2 0 0 8}$ & 18,56 & 12,18 & 8,84 & 24,45 & 21,18 & 23,53 \\
$\mathbf{2 0 0 9}$ & 18,31 & 11,95 & 8,84 & 23,96 & 20,88 & 23,76 \\
$\mathbf{2 0 1 0}$ & 19,77 & 12,70 & 9,56 & 25,99 & 22,72 & 24,95 \\
$\mathbf{2 0 1 1}$ & 20,13 & 12.98 & 9,70 & 26,5 & 22,79 & 26,02 \\
\hline
\end{tabular}

Fonte: IPEADATA

Após a visualização do gráfico 3 logo abaixo, é possível demonstrar a interpretação dos parâmetros que dão origem a função Expectativa de Vida.

O modelo dispõe de uma variável independente, e outra dependente, conforme equação: $\mathbf{Y}=\mathbf{a}+\mathbf{b X 1}$.

Onde:

$\mathrm{Y}=$ Expectativa de Vida do brasileiro

$\mathrm{a}=$ É o coeficiente linear

$\mathrm{b}=$ É o coeficiente angular, indicando o efeito da variável PIBpc sobre a Expectativa de Vida (EV).

Gráfico 1 - Representação Gráfica da Correlação (diagrama de dispersão de pontos ou Scarrtterplot, Brasil - Expectativa de Vida x PIB per capita).

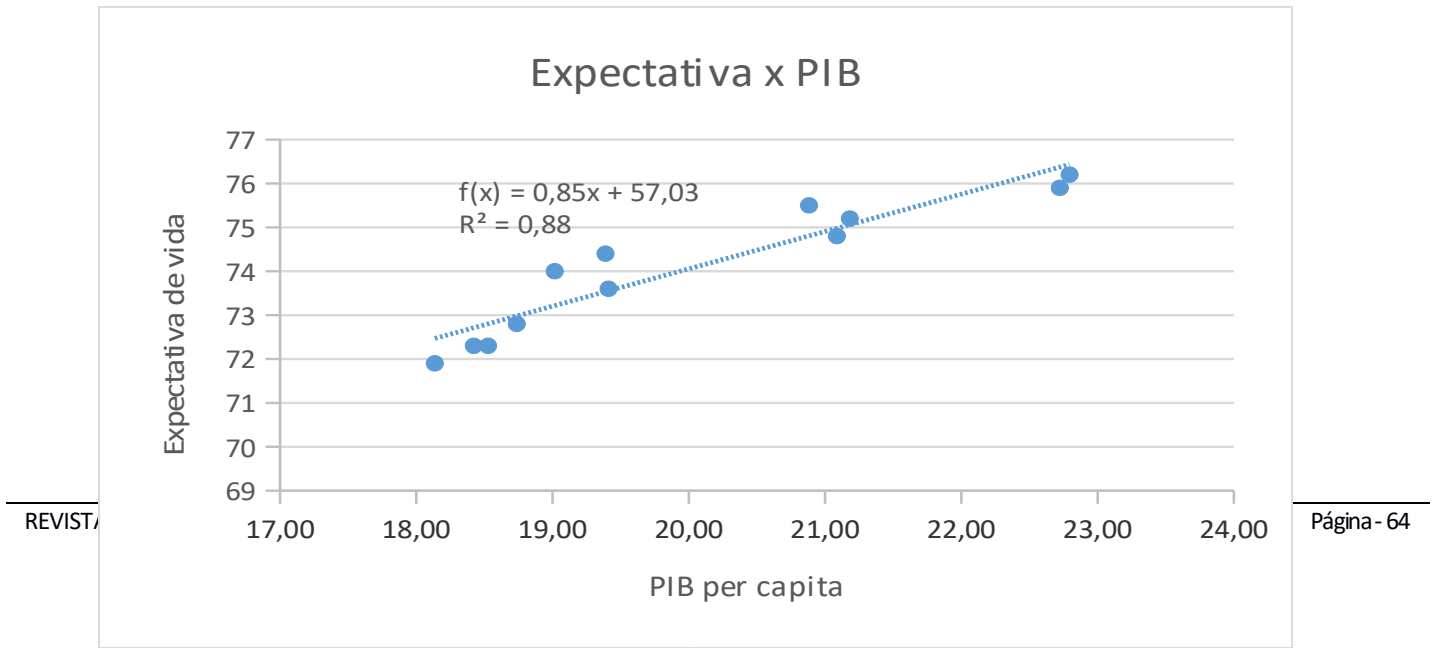


Os dados apresentaram para um modelo de regressão linear simples com a variável dependente EV, em função do PIBpc, um $\mathrm{R}^{2}=0,9063$, mostrando que a EV é explicada em 90,63\% dos casos pelo PIBpc.

\section{EV $=58,4088+0,7969$. PIBpc}

A tabela ANOVA, objetiva descrever se o conjunto das variáveis explicativas tem influência sobre a variável dependente. Isso difere do uso do teste " $t$ ", que testa individualmente a significância dos coeficientes estimadores da regressão.

Segundo Pocinho, (2009) p 74 "O teste F é feito através de uma análise de variância, que separa a variabilidade devido aos "tratamentos" [...] da variabilidade residual, isto é, devido ao acaso. Para aplicar o teste $\mathrm{F}$ é preciso fazer uma série de cálculos, [...].

Para aplicação do teste de Ficher (teste F), a tabela ANOVA.foi analisada. Esse teste mostra se as variáveis em conjunto explicam o comportamento da variável dependente - EV.

O resultado calculado de $\mathrm{F}=96,78$ com um nível de significância Sig 0,0000. O valor tabelado de F para 1 e 10 graus de liberdade é $\mathrm{F}=4,96$, resultante do cruzamento da primeira coluna com a décima linha da tabela F.

Dessa forma, Fcal>Ftab $(96,78>4,96)$, a hipótese de que não há relação entre as variáveis EV e PIBpc, foi rejeitada. Esse resultado demonstra que existe uma probabilidade, menor do que 3,22\% de que F tão alto tenha ocorrido apenas ao acaso. Após que se constatar que o PIBpc - Variável Independente (VI) tem influencia sobre a EV, passamos para o teste " $\mathrm{t}$ " de Student. O objetivo agora é confirmar estatisticamente essa relação, uma vez que no modelo linear simples o teste "t" mede o efeito individual dos parâmetros, equivalendo ao teste $\mathrm{F}$, por existir uma variável independente - PIBpc.

Consultando-se a tabela do teste "t", o valor crítico para 10 graus de liberdade com um nível de significância de 5\% foi de t.tab =1,81 (unilateral) e 2,23(bilateral), demonstrou-se que os valores acima foram menores que o calculado tcal $=41,7028$ e tcal 9,8377, rejeitando-se a hipótese nula.

No que se refere ao intervalo de confiança, isso significa que existe no mínimo $95 \%$ de chance dos valores da constante de do PIBpc pertencerem ao intervalo de 55,288 a 61,529 e 0,616 a 0,977 , respectivamente.

Demonstrou-se que zero não está presente nesse intervalo, o que confirma a conclusão do teste "t" de Student (rejeitamos a Ho =0), com uma margem de erro de no máximo 5\%.

Diante do que foi apresentado verifica-se que a regressão representa bem o modelo da função $\mathrm{EV}$, com os testes $\mathrm{F}$ e t apresentando ótimos resultados, bem com um $\mathrm{R}^{2}=90,6 \%$. 


\section{BRASIL EXPECTATIVA DE VIDA (E.V.) e COEFICIENTE DE GINI. (CGINI)}

Em um segundo momento, utilizando-se dos mesmos recursos expostos acima, diferenciando apenas nas relações com as variáveis. Nesse caso, foi definido o Coeficiente de Gini como a Variável Independente e a Expectativa de Vida (EV) como a Variável Dependente.

Em uma segunda etapa foram dispostos os dados da tabela (1) Expectativa de Vida e Índice de Gini tabela (12), que resultou em um gráfico utilizando a planilha do software EXCEL, foi possível observar a função gerada, conforme a tabela e o gráfico abaixo.

Tabela 4 Índice de GINI: 2000 - 2011.

\begin{tabular}{|l|l|l|l|l|l|l|}
\hline & Brasil & Norte & Nordeste & Sudeste & Sul & C. Oeste \\
\hline $\mathbf{2 0 0 0}$ & 0,597 & 0,598 & 0,612 & 0,575 & 0,564 & 0,621 \\
\hline $\mathbf{2 0 0 1}$ & 0,596 & 0,565 & 0,600 & 0,547 & 0,568 & 0,598 \\
\hline $\mathbf{2 0 0 2}$ & 0,589 & 0,565 & 0,595 & 0,563 & 0,529 & 0,595 \\
\hline $\mathbf{2 0 0 3}$ & 0,583 & 0,543 & 0,585 & 0,557 & 0,531 & 0,580 \\
\hline $\mathbf{2 0 0 4}$ & 0,572 & 0,541 & 0,583 & 0,542 & 0,522 & 0.572 \\
\hline $\mathbf{2 0 0 3}$ & 0,583 & 0,543 & 0,585 & 0,557 & 0,531 & 0,580 \\
\hline $\mathbf{2 0 0 4}$ & 0,572 & 0,541 & 0,583 & 0,542 & 0,522 & 0.572 \\
\hline $\mathbf{2 0 0 5}$ & 0,570 & 0,530 & 0,571 & 0,543 & 0,515 & 0,577 \\
\hline $\mathbf{2 0 0 6}$ & 0,563 & 0,522 & 0,573 & 0,537 & 0,506 & 0,562 \\
\hline $\mathbf{2 0 0 7}$ & 0,560 & 0,535 & 0,564 & 0,523 & 0,505 & 0,574 \\
\hline $\mathbf{2 0 0 8}$ & 0,546 & 0,510 & 0,558 & 0,515 & 0,494 & 0,545 \\
\hline $\mathbf{2 0 0 9}$ & 0,540 & 0,522 & 0,558 & 0,511 & 0,491 & 0,560 \\
\hline $\mathbf{2 0 1 0}$ & 0,536 & 0,543 & 0,555 & 0,517 & 0,480 & 0,546 \\
\hline $\mathbf{2 0 1 1}$ & 0,531 & 0,535 & 0,544 & 0,472 & 0,501 & 0,537 \\
\hline
\end{tabular}

Fonte: IPEADATA e IBGE

* Os valores para anos de 2000 e 2011, são do censo (IBGE)

Gráfico 2 - Representação Gráfica da Correlação de dispersão de pontos ou Scartterplot, Brasil - Expectativa de vida x Índice de Gini.

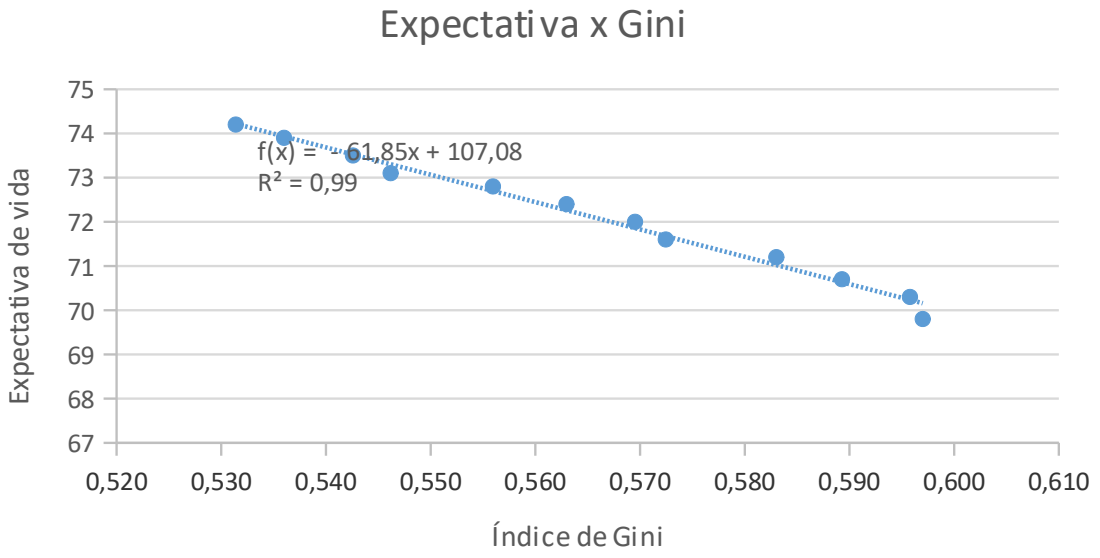

O gráfico 4 acima, retrata uma função linear de relação inversa entre as variáveis, o que é 
esperado, uma vez que o coeficiente de Gini varia 0 e 1 , sendo 0 uma distribuição de renda perfeita. Quanto mais próximo a 1, maior é a desigualdade na distribuição da renda, =interferindo negativamente na EV. Países com uma distribuição perfeita teriam um menor coeficiente de Gini e uma maior Expectativa de vida, vice e versa

Das tabelas que seguem pode-se extrair a seguinte função:

$$
\text { EV }=107,0824-61,8518 C G i n i .
$$

O R $\mathrm{R}^{2}$ 0,9882 demonstra alto poder explicativo da variável independente sobre a variável dependente. Quanto ao teste " $t$ ", observa-se que o $t$ calculado $=88,9507$ (coeficiente) e 29,0605 (C. Gini) foram maiores que o " $t$ " tabelado de 2,23 e 1,81, demonstrando que os coeficientes da regressão são estatisticamente significantes.

Em seguida foi realizado o teste F para comprovar se todos os coeficientes da regressão, em conjunto, eram significantes estatisticamente. O resultado foi Fcal $(844,51)>$ Ftab $(4,96)$, rejeitando a hipótese $b=0$.

ANOVA

Tabela 5 Resultados para o teste F(Fisher) - Brasil

\begin{tabular}{llllll}
\hline & Gl & SQ & MQ & F & F de significação \\
\hline Regressão & 1 & 22,47635437 & 22,47635437 & 844,5133815 & $5,42794 \mathrm{E}-11$ \\
Resíduo $=$ & 10 & 0,266145627 & 0,026614563 & & \\
Total & 11 & 22,7425 & & & \\
\hline
\end{tabular}

\section{AS REGIÕES}

Pelo estudo realizado com o Brasil, foi aplicado o modelo nas regiões brasileiras com a finalidade de conhecer o comportamento do PIB per capita e o grau de concentração de renda, por meio o Coeficiente de Gini. Em todas as regiões utilizou-se o PIB per capita como variável independente obtendo-se um $\mathrm{R}^{2}$ significante de 0,9778 (Norte), 0,9641(Centro-Oeste), 0,9448 (Nordeste), 0,8803(Sul) e 0,8583 (Sudeste).

Quando o Coeficiente de Gini, que mede o grau de concentração de renda, para explicar a Expectativa de Vida, foi utilizado, somente à região Norte, apresentou um baixo $\mathrm{R}^{2}$, ficando em 0,5593. As demais regiões os valores de $\mathrm{R}^{2}$ foram significativos, compreendidos entre 0,9755 (NE) e $0,8278(S)$, conforme tabela abaixo. 
Tabela 6 Resultados para o coeficiente de determinação $\left(\mathrm{R}^{2}\right)$

\begin{tabular}{cccccc}
\hline \hline Regiões & Norte & Nordeste & Sudeste & Sul & Centro Oeste \\
$\mathrm{R}^{2}$ & 0,5593 & 0,9448 & 0,8583 & 0,8803 & 0,9641 \\
\hline
\end{tabular}

Em seguida foi aplicado o teste $\mathrm{F}$, revelando para todas as regiões que as variáveis em conjunto: PIBpc e CGini apresentam, uma excelente influencia sobre a variável dependente (Expectativa de Vida). Em todos os casos Fcal $>$ Ftab.

Tabela 7 Resultados para o teste F (Fischer), Regiões do Brasil.

\begin{tabular}{c|c|c|c|c}
\hline \multirow{2}{*}{ Regiões } & \multicolumn{2}{|c|}{ CALCULADO F } & \multicolumn{2}{c}{ TABELADO F } \\
\hline \multirow{3}{*}{ Norte } & PIBpc & CGINI & PIBpc & CGINI \\
Nordeste & 441.684 & 12.695 & 4,96 & 4,96 \\
Sudeste & 171.162 & 398.941 & 4,96 & 4,96 \\
Sul & 269,088 & 82,535 & 4,96 & 4,96 \\
Centro Oeste & 73.560 & 48,090 & 4,96 & 4,96 \\
\hline
\end{tabular}

O valor crítico (tabelado) de F, para 1 e 10 graus de liberdade no numerador e denominador respectivamente (verificar cruzamento da primeira coluna com a décima linha para nível de significância de 5\%, na tabela de valores críticos de $\mathrm{F}$ ) é de $\mathrm{F}=4,96$. Com valores Fcal $>$ Ftab, rejeitouse a hipótese de efeito nulo Ho $=0$ e aceitando-se a hipótese alternativa no nível de significância $5 \%$.

Tabela 8 Resultados do teste t(Studant): Regiões (Nortes, Nordeste, Sudeste, Sul, Centro Oeste)

\begin{tabular}{|c|c|c|c|c|c|}
\hline \multicolumn{2}{|c|}{ Regiões } & \multicolumn{2}{|c|}{ Calculado (t) } & \multicolumn{2}{|c|}{$\begin{array}{l}\text { TABELADO (T) } \\
\text { Teste Unicaudal. }\end{array}$} \\
\hline & & PIBpc & CGINI & PIBpc & CGINI \\
\hline \multirow[t]{2}{*}{$\mathbf{N}$} & $\mathrm{Ce}$ & 146,203 & 17,620 & 1,81 & 1,81 \\
\hline & $\mathrm{Vx}$ & 21,016 & $-3,563$ & 1,81 & 1,81 \\
\hline \multirow[t]{2}{*}{$\mathrm{Ne}$} & $\mathrm{CE}$ & 62,609 & 26,746 & 1,81 & 1,81 \\
\hline & $\mathrm{Vx}$ & 13,082 & $-7,272$ & 1,81 & 1,81 \\
\hline \multirow[t]{2}{*}{ Se } & $\mathrm{CE}$ & 32,284 & 26,746 & 1,81 & 1,81 \\
\hline & $\mathrm{Vx}$ & 7,783 & $-7,272$ & 1,81 & 1,81 \\
\hline \multirow[t]{2}{*}{$\mathbf{S}$} & $\mathrm{Ce}$ & 28,617 & 26,883 & 1,81 & 1,81 \\
\hline & $\mathrm{Vx}$ & 8,576 & $-6,934$ & 1,81 & 1,81 \\
\hline \multirow{2}{*}{$\begin{array}{c}\text { Centro } \\
\text { Oeste }\end{array}$} & $\mathrm{Ce}$ & 114,247 & 36,409 & 1,81 & 1,81 \\
\hline & $\mathrm{Vx}$ & 16,403 & $-9,084$ & 1,81 & 1,81 \\
\hline
\end{tabular}

O valor tabelado (crítico) para 10 graus de liberdade e nível de significância de 5\%, no 
caso do teste $t$ unilateral é de $t$ tab $=1,81$ e de ttab=2,23 (bilateral). Portanto, a hipótese nula foi rejeitada e a hipótese alternativa foi aceita para os valores calculados, os valores foram maiores que 0 . Com valores tcal $>$ ttab.

Os resultados do modelo de regressão que constaram ao longo dessa análise, apresentaram excelentes resultados para o Brasil e para todas as regiões.

Os dados identificados pela correlação de Person foram bastante positivos pelos valores apresentados, verificou-se que tanto analisando o Brasil, como as regiões (Norte, Nordeste, Centro-Oeste, Sul e Sudeste) existe uma forte correlação positiva entre Expectativa de Vida e PIB per capita, cujos valores variaram entre 0,926 e 0,988 .

Quanto a correlação R, entre Expectativa de Vida e Coeficiente de Gini, que varia entre 0 e 1 , todos apresentaram uma boa correlação negativa, pois mais próximo a 0 , implicaria em uma maior Expectativa de Vida. A região Norte foi a que apresentou um Coeficiente de Gini mais distorcido, - 0,747, se comparado com os das outras regiões e do Brasil, que variaram entre (0,909 a -0,994), os R's (Correlação) para o PIB apresentaram ótimos resultados para o PIB per capita, valores entre 0,926 e 0,988 .

Os resultados observados na pesquisa para o teste $\mathrm{F}$ para o Brasil e todas as regiões, verifica-se que "F calculado" foi maior que "F tabelado" ou Fcal > Sig, o mesmo foi verificado para o teste $t$, os valores do $t$ calculo foi maior que $t$ tabelado, de que forma que as variáveis em conjunto são significantes estatisticamente, e por isso, rejeitou-se a hipótese nula aceitando-se a hipótese alternativa com erro máximo de $5 \%$.

O que se concluir e que as variáveis independentes PIB per capita e Índice de Gini são bons parâmetros para explicar a variável dependente expectativa de vida.

\section{CONSIDERAÇÕES FINAIS}

O Brasil possui um grande potencial para alcançar um nível de qualidade de vida, semelhante ao observado em países desenvolvidos, em algumas décadas. Para tanto, não basta que o governo realize investimentos na economia, já que o crescimento econômico não determina necessariamente o desenvolvimento social. Dessa maneira, deve-se voltar a atenção para fatores que promovam equidade social, como aumento da oferta de emprego, qualificação profissional e a melhora dos níveis de saúde da população. Apenas através de uma mudança estrutural e continuada desses fatores, o país alcançará um desenvolvimento social permanente e adequado.

A relação existente entre crescimento econômico e desenvolvimento social é significativa, principalmente quando se observa os dados brasileiros de PIB per capita, Coeficiente de Gini e Expectativa de Vida. Esses indicadores alcançaram significativa melhora 
no período de 2000 a 2011.

O crescimento econômico foi acompanhado de uma significativa elevação do PIB per capita, que indica uma melhora no poder aquisitivo da população brasileira. Outro indicador social relevante, que obteve bons resultados, evidenciando a melhoria das condições de vida em termos de rendimentos, expectativa de vida, saúde e educação, foi o Coeficiente de Gini

O comportamento do coeficiente de Gini, apresentou melhora, passando de 0,597 em 2000 para 0,531 em 2011. Esse resultado esboça uma diminuição, ainda incipiente, da desigualdade social no país. É imprescindível ressaltar a diminuição da população que vive em extrema pobreza: uma queda equivalente a 9 pontos percentuais. O caminho a ser percorrido para alcançar um patamar de desenvolvimento social e econômico satisfatório deverá ser continuo a fim de não haver perdas nas conquistas dos últimos anos.

A pesquisa contou com um estudo econométrico utilizando para tanto um modelo de regressão linear simples, para o Brasil e as cinco regiões no período de 2000 a 2011.

O modelo analisou se as variáveis: PIB per capita e Índice de Gini são bons parâmetros para explicar a expectativa de vida. $\mathrm{O}$ resultado foi positivo para todas as regiões.

O R (coeficiente de correlação) e o R ${ }^{2}$ (coeficiente de determinação) apresentaram ótimos resultados para o PIB per capita e coeficiente de Gini.

Existe uma correlação negativa entre Expectativa de Vida e Coeficiente de Gini, os quais apresentaram uma boa correlação negativa com variação entre $(-0,909$ a $-0,994)$ a região Norte foi a que apresentou um Coeficiente de Gini, - 0,747 mais distorcido, para o PIB per capita apresentaram ótimos resultados valores entre0,926 e 0,988 .

$\mathrm{R}^{2}$ (coeficiente de determinação) apresentou excelente resultado para o PIB per capita, com valores entre $0,858(\mathrm{SE})$ e $0,977(\mathrm{~N})$. Quando o Coeficiente de Gini, que mede o grau de concentração de renda, para explicar a Expectativa de Vida, foi analisado, somente à região Norte, apresentou um baixo $\mathrm{R}^{2}$, ficando em 0,593 . As demais regiões os valores de $\mathrm{R}^{2}$ foram significativos, compreendidos entre 0,9755 (NE) e 0,8278(S),

Os resultados observados na pesquisa do teste $\mathrm{F}$ para o Brasil e todas as regiões, verificou que o "F calculado" foi maior que "F tabelado" ou Fcal > Sig, o mesmo foi verificado para o teste $t$, os valores do $t$ calculado foram maiores que $t$ tabelado, de que forma que as variáveis em conjunto são significantes estatisticamente, e por isso, foi rejeitada a hipótese nula e aceito a hipótese alternativa, com erro máximono nível de significância de 5\%.

Uma significativa elevação do PIB per capita, indica uma melhora no poder aquisitivo da população brasileira, proporcionando um impacto positivo sobre a expectativa média de vida. 
Da mesma forma a expectativa de vida tende a crescer com a melhora do coeficiente de Gini que se mostrou linear para todas as regiões.

O que se pode concluir com todos esses resultados é que é possível comprovar a eficácia do modelo econométrico na estimativa das variáveis independentes: Índice de Gini e PIB per capita explicando a variável dependente (Expectativa de vida) que foram testados no Brasil e nas cinco regiões brasileiras.

Os resultados do modelo proposto para Brasil e as cinco regiões brasileiras são bastante significativos. O enfoque na correlação da expectativa de vida com: PIB per capita e Índice de Gini, corrobora a hipótese apresentada, contudo, sugere-se que outras pesquisas possam aprofundar o estudo em tela.

\section{REFERÊNCIAS}

GIOVANELLA, Ligia; Scorel, Sarah; LOBATO, Lenaura de Vasconcelos Costa; NORONHA, José Carvalho de; Carvalho, Antônio Ivo de.,Políticas e Sistemas de Saúde no Brasil (online) Rio de Janeiro: Editora Fiocruz, 2012, 2nd, ed rev. 1100 p. Avilable from Scielo Books.Disponível em:

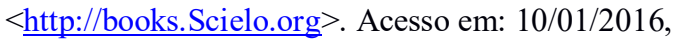

IBGE, Instituto Brasileiro de Geografia e Estatística Disponível em: $<$ http://www.ibge.gov.br/home/geociencias/areaterritorial/principal.shtm $>$. Acesso em: 19/06/2016.

IBGE, Observações sobre a evolução da mortalidade no Brasil: o passado, o presente e perspectivas. Rio de Janeiro, $2010 . \quad$ Disponível em: $<$ http://www.ibge.gov.br/home/estatistica/populacao/tabuadevida/2009/notastecnicas.pdf $>$. Acesso em: 14/01/2016.

IPEADATA, Instituto de Pesquisa Aplicada, Uma década de crescimento com diminuição da desigualdade. Disponível em: $<$ http://www.ipea.gov.br/code2011/chamada2011/pdf/area2/area2artigo32.pdf>. Acesso em: 29/01/2016.

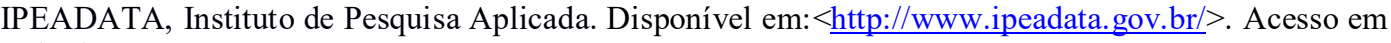
$11 / 03 / 2016$.

IPEADATA, Instituto de Pesquisa Aplicada, Década Inclusiva (2001-2011): Desigualdade, Pobreza e Políticas de

Renda.

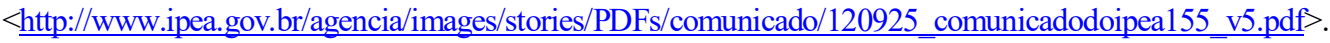

Acesso em: 05/06/2016.

PELEGRINI, Maria Letícia de; CASTRO,Janice Dornelles de , Expectativa de Vida e Gastos Públicos em Saúde Disponível em:<seer.ufrgs.br/AnaliseEconomica/article/download/25879/21542>. Acesso em: 16/03/2016.

POCINHO, Margarida, Estatística Volume I Teoria e exercícios passo-a-passo, 2009. Disponível em:

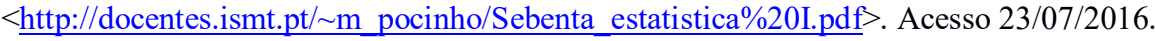

PIACENTI, Carlos Alberto;LIMA, Jandir Ferreira de; STAMM, Cristiano; PIFFER, Moacir; ALVES, Lucir Reinaldo, A Dinâmica Dos Estados Da Região Sul Do Brasil, 2002. Disponível em: <files.luciralves.com/.../A\%20dinâmica\%20dos\%20estados\%20da\%20regi...>. Acesso em: 17/06/2016.

SILVA, L.G.De.C E., A TÁBUA DE MORTALIDADE DO RPPS DO ESTADO DE SÃO PAULO, 2007. Disponível $\quad \mathrm{em}:<$ http://www.abep.nepo.unicamp.br/encontro2010/docs pdf/tema 9/abep2010_1966.pdf $>$. Acesso em: 10/03/2016.

SILVA, L.G.De E.C.,Nota Técnica sobre a Metodologia adotada pelo Ministério da Previdência Social na Extrapolação das Tábuas de Mortalidade IBGE para as idades acima de 80, anos, 2008. Disponível. < <http://www.mtps.gov.br/images/RPPS/LegislacaodosRPPS/NOTATECNICA.pdf $>$. Acesso em:09/03/2016. 